\title{
Lymphatic Interrupted: Do We Really Understand the Risks and Consequences?
}

\author{
Elizabeth A. Mittendorf, MD and Kelly K. Hunt, MD \\ Department of Surgical Oncology, Unit 444, The University of Texas M. D. Anderson Cancer Center, Houston, TX
}

Lymphedema remains one of the most feared complications of breast cancer therapy, yet at the same time, is arguably one of the least well understood. Review of the literature reveals inconsistencies in defining and measuring this condition, as well as in understanding the extent of lymphedema that is clinically significant. The true incidence of lymphedema is widely debated, as are the risk factors leading to its development. The surgical removal of lymph nodes and disruption of lymphatic channels during dissection of the axilla clearly contribute, but there are other variables that play a role, including treatment-related variables such as use of radiation therapy, and patientrelated variables. Some of the most frequently reported patient-related factors are increasing age, higher body mass index, and weight gain. Although infection and injury have been reported to be inciting events in the development of lymphedema, these relationships are less clear. ${ }^{1,2}$

Attempting to address some of the shortcomings of the existing literature, in the current issue of Annals of Surgical Oncology, Yen et al. report their findings of risk factors for development of lymphedema in a population-based study of breast cancer patients over 65 years of age. Patients were identified through Medicare claims which were queried along with state tumor registries to extract clinicopathologic data. The presence of lymphedema was a self-reported measure and, at a median of 48 months postoperatively, $193(14.4 \%)$ women reported evidence of lymphedema. After controlling for tumor size, histology, type of surgery, and adjuvant therapies, the investigators found that removal of more than five lymph nodes and presence of lymph node metastases were independent predictors of developing lymphedema. These data and the

(C) Society of Surgical Oncology 2009

Published Online: 8 April 2009

E. A. Mittendorf, MD

e-mail: eamitten@mdanderson.org authors' conclusions raise several issues that warrant further discussion as we seek to understand the relative contributions of disease and therapeutic intervention in breast cancer outcomes.

One thing that we can all agree on is that there is no consensus on what quantitatively constitutes lymphedema. Volume displacement or circumferential measurements of the arm have been the most commonly employed methods for assessing lymphedema; however, these methods have not been standardized, and defining lymphedema based on specific measurements may not be clinically relevant. Yen and colleagues suggest that a potential weakness of their study was the fact that lymphedema was a self-reported outcome by the study participants. In fact, symptom assessment may be an underutilized tool for identifying patients with lymphedema. Patient's may report that their arm feels heavy before lymphedema is significant enough to lead to stretching of the skin and subcutaneous tissue that would be required to cause a change in volume or circumferential measurement. Armer et al. have demonstrated that there is a high predictive ability between objective measures of extremity swelling and the selfreported outcome of arm heaviness. ${ }^{3}$ A recent study by Norman et al. also demonstrated that differences in selfreported arm size and symptoms can be early signs of progressing lymphedema. ${ }^{4}$ Subjective limb assessment, including asking if their arm feels heavy, is an important measure, and patients reporting arm heaviness should be referred for appropriate evaluation by a physical therapist with expertise in lymphedema management.

With respect to factors predisposing for the development of lymphedema, Yen and colleagues determined that only removal of more then five lymph nodes and presence of lymph node metastases were significant. These findings have important implications given current breast cancer treatment recommendations. Sentinel lymph node dissection (SLND) is now routinely utilized for axillary staging 
in patients with early-stage breast cancer. Several multicenter trials and large single-institution experiences have reported that the median number of lymph nodes removed during SLND ranges between 2 and 3. ${ }^{5-7}$ The data reported by Yen et al. that removal of more than five lymph nodes is what predisposes women to increased risk of lymphedema might lead one to conclude that there is no increased risk of lymphedema in patients undergoing SLND with fewer than five lymph nodes recovered. We know from the reports of the American College of Surgeons Oncology Group (ACOSOG) sentinel lymph node trials and the Axillary Lymphatic Mapping against Nodal Axillary Clearance (ALMANAC) trial that this is not the case. Lymphedema rates of approximately $7 \%$ and $5 \%$ at 1 year after surgery have been reported for the ACOSOG Z0010 and ALMANAC trials, respectively. ${ }^{2,8}$ In studies reporting on the development of lymphedema, there is significant variability in the time between surgery and the development of arm symptoms; therefore it is anticipated that the rates in these trials may increase with longer follow-up. In the population-based study by Yen et al., 319 patients underwent SLND alone and 7\% developed lymphedema. This rate of lymphedema was similar to the rate of $5.7 \%$ that was reported in the $20 \%$ of their cohort that did not undergo any axillary surgery. These percentages must be interpreted with caution, as the number of lymph nodes removed, regardless of the axillary procedure, was highly variable. For example, in the patients who reportedly had no axillary surgery, the authors reported that the number of lymph nodes evaluated ranged between 0 and 15 . This highlights the difficulty of using data extracted from large databases where the investigators do not have the ability to review the primary source documents for accuracy. Recognizing these limitations, the authors suggest that it is important to counsel patients undergoing SLND regarding their risk of developing lymphedema. While this is certainly an important part of preoperative education and informed consent, it is important to remember that the sentinel lymph node procedure is performed for staging and that the accuracy of this staging procedure may be impacted if the surgeon does not remove all of the sentinel nodes. The number of sentinel nodes will vary from patient to patient. Several investigators have attempted to determine the number of sentinel lymph nodes (SLN) that need to be removed for accurate staging and have reported that four or five nodes is enough. In a recent study from our institution evaluating over 775 patients with a positive SLN, we found that in $75 \%$ of patients the first SLN removed was the one containing metastatic disease. In $91 \%$ of patients, the first metastasis was identified in one of the first two lymph nodes removed. Removal of five SLNs allowed for recovery of $>99 \%$ of all positive SLNs. These data suggest that, in the majority of patients, removing up to five SLNs will be adequate to appropriately stage the axilla; however, additional clinical and pathologic factors may impact this number and if additional blue or hot SLNs remain after five are excised, then removal of more lymph nodes may be required. ${ }^{7}$ With respect to the risk of lymphedema following SLND, it may not be the number of nodes that are removed but the extent of the dissection and the number of lymphatic channels that are disrupted during the recovery of the SLNs that predisposes a patient to the risk of developing this condition.

The second risk factor reported by Yen et al. to predict lymphedema was the presence of lymph node metastases. In their discussion, the authors speculate that this may be due to the addition of adjuvant therapies, including radiation; however they argue against this for three reasons: (1) they were unable to identify patients that underwent axillary radiation, as radiation therapy billing codes do not differentiate between treatment fields; (2) axillary radiation is not routinely used in the adjuvant setting for women with early-stage breast cancer; and (3) their statistical analysis did not demonstrate an association between radiation therapy and development of lymphedema. Again, the reader should be cautioned since the authors note that their ability to accurately determine the extent of radiation was significantly limited. As per current guidelines, patients with node-positive disease may receive adjuvant radiation of the level III axilla and supraclavicular fossa as a component of their treatment. ${ }^{9}$ It is also known that a significant component of the level I and II axilla is within the standard tangential breast irradiation fields utilized for early-stage breast cancer patients treated with breast radiation following lumpectomy. ${ }^{10}$ It is not a stretch to assume that such treatment can cause radiation-induced fibrosis leading to lymphatic obstruction and subsequent lymphedema.

As clearly demonstrated in this study by Yen et al., lymphedema remains a potential morbidity of breast cancer therapy regardless of our attempts to decrease the extent of surgical intervention. To address the many inconsistencies in our current understanding of the risk of developing lymphedema, clinicians must reach a consensus on the appropriate measures and timing of those measures. Welldesigned clinical trials incorporating those measures will then be required to better determine the incidence, risk factors, and appropriate treatment of lymphedema. Currently, one cooperative group trial (CALGB 70305: A Randomized Study to Prevent Lymphedema in Women Treated for Breast Cancer) is randomizing patients undergoing axillary lymph node dissection to one of two arms: education only, or an education and treatment arm that will include physical-therapy-focused interventions. We would encourage surgeons caring for breast cancer patients to consider enrolling their patients in this trial. Until the time 
that such studies are completed, surgeons should continue to counsel their breast cancer patients about the risk of lymphedema with surgical treatment and the need for early intervention to minimize the extent of arm swelling that may occur.

\section{REFERENCES}

1. McLaughlin SA, Wright MJ, Morris KT, et al. Prevalence of lymphedema in women with breast cancer 5 years after sentinel lymph node biopsy or axillary dissection: patient perceptions and precautionary behaviors. J Clin Oncol. 2008;26(32):5220-6.

2. Wilke LG, McCall LM, Posther KE, et al. Surgical complications associated with sentinel lymph node biopsy: results from a prospective international cooperative group trial. Ann Surg Oncol. 2006;13(4):491-500.

3. Armer JM, Radina ME, Porock D, Culbertson SD. Predicting breast cancer-related lymphedema using self-reported symptoms. Nurs Res. 2003;52(6):370-9.

4. Norman SA, Localio AR, Potashnik SL, et al. Lymphedema in breast cancer survivors: incidence, degree, time course, treatment, and symptoms. J Clin Oncol. 2009;27(3):390-7.
5. Krag DN, Anderson SJ, Julian TB, et al. Technical outcomes of sentinel-lymph-node resection and conventional axillary-lymphnode dissection in patients with clinically node-negative breast cancer: results from the NSABP B-32 randomised phase III trial. Lancet Oncol. 2007;8(10):881-8.

6. Lucci A, McCall LM, Beitsch PD, et al. Surgical complications associated with sentinel lymph node dissection (SLND) plus axillary lymph node dissection compared with SLND alone in the American College of Surgeons Oncology Group Trial Z0011. J Clin Oncol. 2007;25(24):3657-63.

7. Yi M, Meric-Bernstam F, Ross MI, et al. How many sentinel lymph nodes are enough during sentinel lymph node dissection for breast cancer? Cancer. 2008;113(1):30-7.

8. Mansel RE, Fallowfield L, Kissin M, et al. Randomized multicenter trial of sentinel node biopsy versus standard axillary treatment in operable breast cancer: the ALMANAC Trial. $J$ Natl Cancer Inst. 2006;98(9):599-609.

9. Buchholz TA. Radiation therapy for early-stage breast cancer after breast-conserving surgery. N Engl J Med. 2009;360(1): 63-70.

10. Schlembach PJ, Buchholz TA, Ross MI, et al. Relationship of sentinel and axillary level I-II lymph nodes to tangential fields used in breast irradiation. Int J Radiat Oncol Biol Phys. 2001; 51(3):671-8. 